\title{
Numerical Simulation of Solid Tumor Blood Perfusion and Drug Delivery during the "Vascular Normalization Window" with Antiangiogenic Therapy
}

\author{
Jie Lv, ${ }^{1}$ Jin-feng Cao, ${ }^{1}$ Yan Cai, ${ }^{1}$ Yu Zhou, ${ }^{1}$ Quan Long, ${ }^{2}$ \\ Wei Yao, ${ }^{1}$ and Shi-xiong $X \mathbf{u}^{1}$ \\ ${ }^{1}$ Department of Mechanics and Engineering Science, Fudan University, Shanghai 200433, China \\ ${ }^{2}$ Brunel Institute for Bioengineering, Brunel University, Middlesex, Uxbridge UB8 3PH, UK
}

Correspondence should be addressed to Shi-xiong Xu, xusx@hotmail.com

Received 19 January 2011; Revised 1 March 2011; Accepted 31 March 2011

Academic Editor: Jürgen Geiser

Copyright (C) 2011 Jie Lv et al. This is an open access article distributed under the Creative Commons Attribution License, which permits unrestricted use, distribution, and reproduction in any medium, provided the original work is properly cited.

To investigate the influence of vascular normalization on solid tumor blood perfusion and drug delivery, we used the generated blood vessel network for simulations. Considering the hemodynamic parameters changing after antiangiogenic therapies, the results show that the interstitial fluid pressure (IFP) in tumor tissue domain decreases while the pressure gradient increases during the normalization window. The decreased IFP results in more efficient delivery of conventional drugs to the targeted cancer cells. The outcome of therapies will improve if the antiangiogenic therapies and conventional therapies are carefully scheduled.

\section{Introduction}

Angiogenesis, the growth of a network of blood vessels, plays a significant role in tumor's growth, invasion, and metastasis. Therefore, blocking angiogenesis could be a strategy for arresting tumor growth [1]. As the features of angiogenesis inhibitors are of low toxicity and have less acquired resistance, it comes to a new strategy to suppress tumor growth by restraining angiogenesis. The widely held view is that the antiangiogenic therapies can destroy the tumor vasculature, thereby depriving the tumor of oxygen and nutrients. When administered as single agents, antiangiogenic therapies have produced modest objective responses in clinical trials, but they have not obtained a long-term effect. However, several preclinical and clinical studies have shown that antiangiogenic therapies 


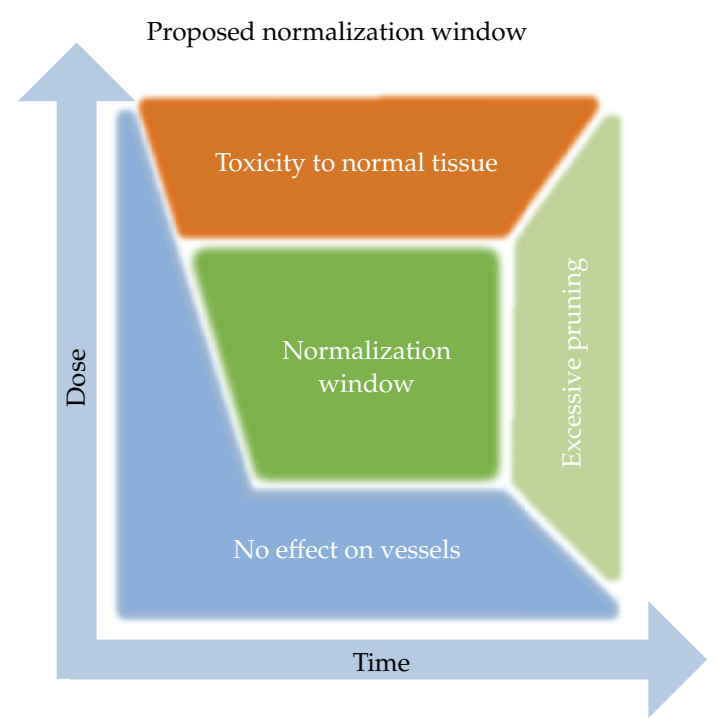

Figure 1: Proposed effect of drug dose and schedule on tumor vasculature normalization window [2].

improve the outcome of cytotoxic therapies. This is paradoxical. Based on that, Jain [2] proposed a new hypothesis that the antiangiogenic therapies can transiently "normalize" the abnormal structure and function of tumor vascular and microenvironment (Figure 1). There is a "vasculature normalization window" during antiangiogenic therapy. This view has been supported by clinical data.

The vasculature of solid tumors is structurally and functionally different from normal blood vessels [3]. They are leaky, tortuous, dilated, and saccular. Branches are irregular, and vessel diameters are uneven. In addition, the vessel wall has abnormal structure. The endothelial cells have aberrant morphology. They are loosely attached, and usually there are gaps. The basement membrane has diverse thickness with broken or missing pieces.

In normal tissues, interstitial fluid pressure (IFP) is close to zero; however, IFP is elevated in tumors in patients. The high IFP poses a strong barrier to the delivery of drugs into tumors and interstitium [4]. There are two other characteristics of the abnormal tumor microenvironment-hypoxia and acidosis [5-7]. One of the main functions of vasculature system is to provide cells with nutrition and oxygen and take away the waste of metabolism at the same time. However, tumor vasculature does not have the same function. Hypoxia renders tumor cells resistant to both radiation and cytotoxic drugs. Meanwhile, the low $\mathrm{PH}$ value hindered the efficacy of therapies.

Above all of these show that if we could find a way to fix the abnormal tumor vasculature and microenvironment, we would improve the treatment of cancer. Jain [8] showed that antiangiogenic therapy could cause a transient "vascular normalization window" and decrease IFP by changing the tumor sizes, vascular hydraulic permeability, and/or the surface area per unit tissue volume of tumor vessels. Normalization of tumor vasculature and microenvironment results in more efficient delivery of conventional drugs to the targeted cancer cells. The outcome of therapies will improve if the antiangiogenic therapies and conventional therapies are carefully scheduled.

In recent years, several mathematical models used different approaches to improve the understanding of tumor hemodynamics. McDougall et al. (2002) [9] built a two-dimensional 


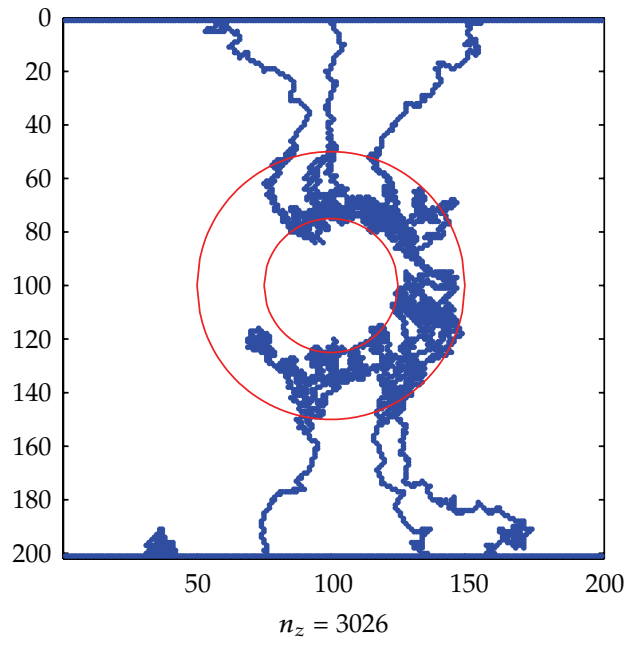

(a)

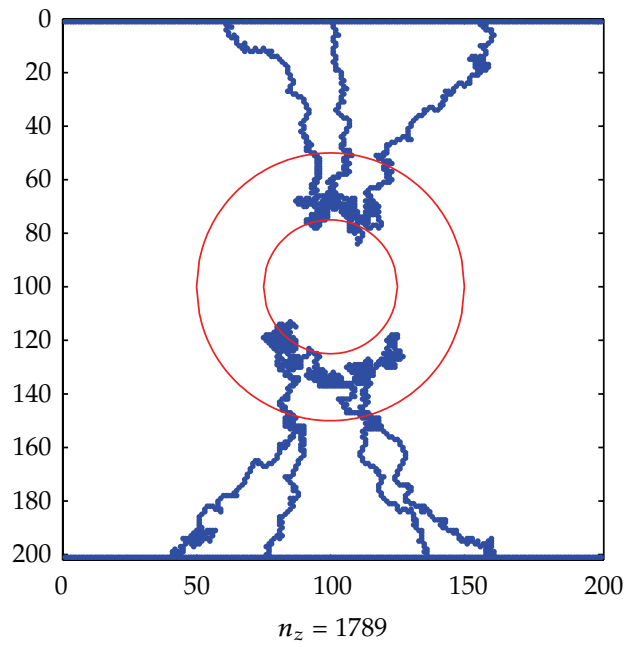

(b)

Figure 2: The vasculature for flow simulations; the blue lines on top and bottom of lattice represent the parent vessels, and the big red circle represents the tumor domain. (a) The tumor vasculature without antiangiogenesis therapy; (b) the tumor vasculature under antiangiogenesis therapy.

capillary network model to study nutrition, oxygen, and drugs transport to the tumor. Tee et al. (2004) [10] created a discrete model to simulate the drug delivery mode under endostatin and the effect on tumor angiogenesis caused by drug clearance. Our research group has done some related work. Gaiping et al. (2006) [11] built the network model under Endostatin using random discrete methods. Wu et al. (2009) [12] simulated tumor blood perfusion based on $3 \mathrm{D}$ angiogenic microvasculature. Here, we use 2D microvasculature built by the group before and simulate the blood perfusion and drug delivery during vasculature normalization.

\section{Blood Perfusion during the "Vasculature Normalization Window"}

We employ a mathematical model to show the effect of the kinetic parameters on tumor blood perfusion; detailed equations and numerical methods can be seen in [12].

In this paper, we used the vascular network generated by our group for the simulation, shown in Figure 2. The actual size is $4 \mathrm{~mm} \times 4 \mathrm{~mm}$, divided into $200 \times 200$ equally spaced grids. Tumor was located in the center of the region, with a radius of $1 \mathrm{~mm}$. The surrounding is normal tissue, and two parent vessels are in the upper and lower bounds, respectively.

During the "vascular normalization window," several parameter values of microenvironment become close to normal tissues [13-16], such as $L_{p}, K_{P}, S / V, \sigma\left(\pi_{v}-\pi_{i}\right)$, and $R$. Here, $L_{p}$ is the vascular wall permeability $(\mathrm{cm} / \mathrm{mmHgs}), K_{P}$ is the interstitial hydraulic conductivity $\left(\mathrm{cm}^{2} / \mathrm{mmHgs}\right), S / V$ is the surface area of vessel wall per unit volume of tissue $\left(\mathrm{cm}^{-1}\right), \sigma\left(\pi_{v}-\pi_{i}\right)$ is oncotic pressure $(\mathrm{mmHg})$, and $R$ is the tumor radius. Our analysis suggests, however, that $\sigma\left(\pi_{v}-\pi_{i}\right)$ is much smaller than the value of normal tissues, even after antiangiogenic therapies. Consequently, oncotic pressure dose not significantly influence the tumor blood perfusion.

The simulation results are shown in Figure 3. We show here that vascular normalization can decrease tumor IFP in a number of ways. The maximum value of IFP dropped from $11.87 \mathrm{mmHg}$ to $4.06 \mathrm{mmHg}$. Furthermore, the phenomenon of high interstitial pressure 


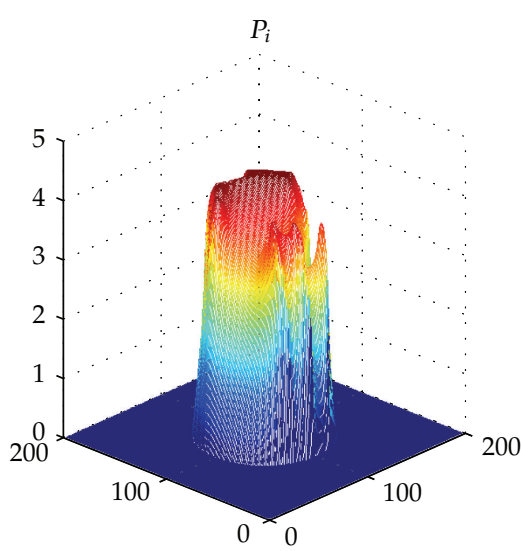

(a) before treatment
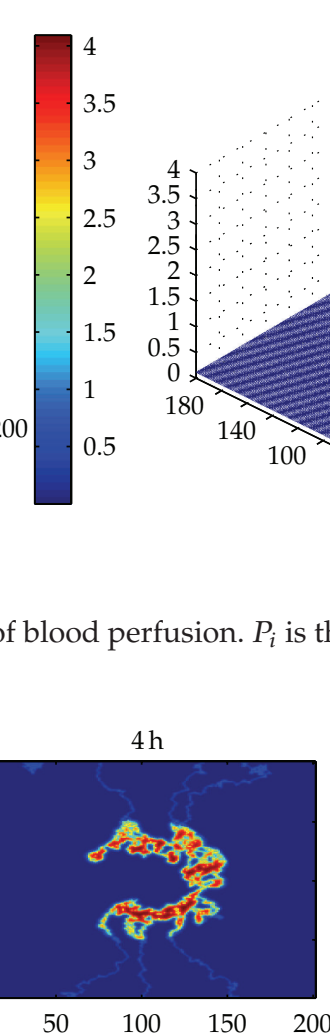

(b)

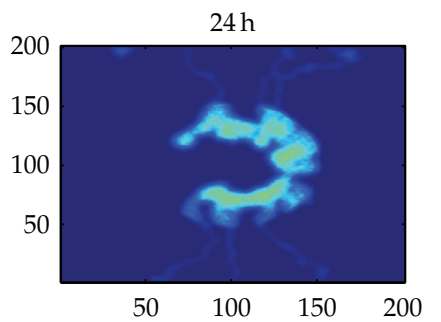

(e)

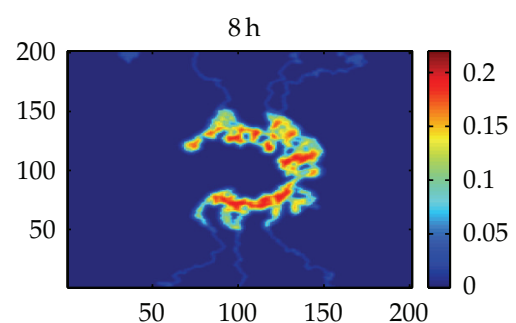

(c)

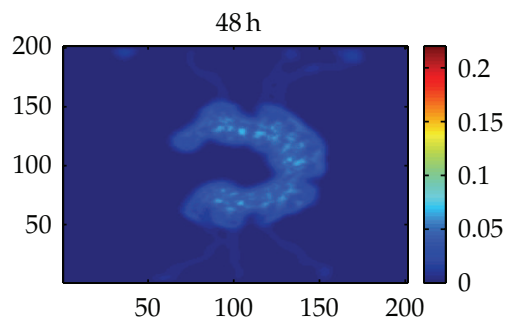

(f)

Figure 4: The simulation result of drug concentration before treatment.

gradient has been greatly improved, except that the boundary of the tumor still has a slightly higher pressure gradient. All of these will benefit the drug delivery and the outcome of conventional therapies.

\section{Drug Delivery during Vascular Normalization}

\subsection{Mathematical Model}

Drugs enter the circulatory system of human body and transport to the tumor microcirculation. To balance the plasma drug concentration is related to the systemic circulation. 


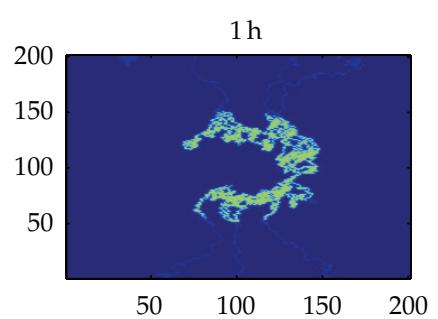

(a)

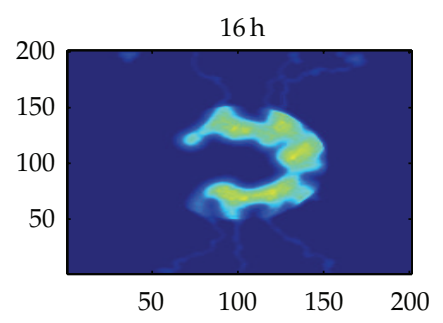

(d)

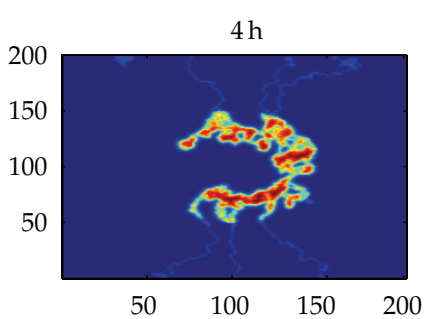

(b)

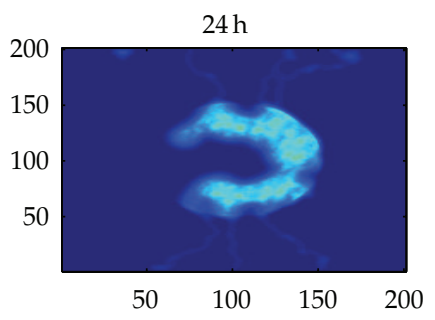

(e)

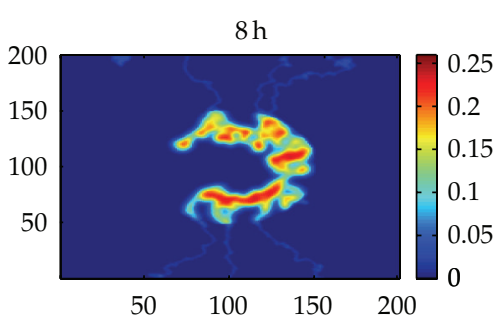

(c)

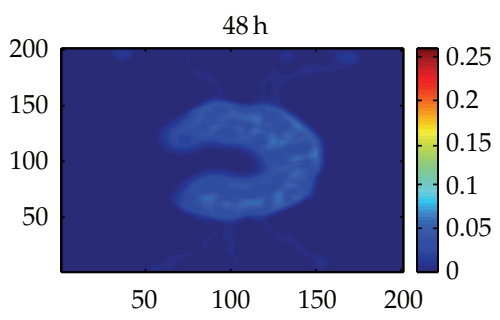

(f)

Figure 5: The simulation result of drug concentration after treatment.

Compared with the scale of the human body, the tumor is small. Therefore, the influence on plasma drug concentration reaching stable state can be neglected. In our model, drugs enter the interstitium through capillary walls. A part of the drugs in the interstitium are absorbed by lymphatic vessels as well as cleared by certain organs. The average drug concentrations in plasma $\left(C_{P}\right)$ and in interstitium $\left(C_{e}\right)$ are represented by the following equations:

$$
\begin{gathered}
\frac{d C_{P}(t)}{d t}=-k e \cdot C_{P}(t)-K \cdot C_{P}+I(t), \\
\frac{d C_{e}(t)}{d t}=K \cdot C_{p}(t)-L \cdot C_{e}(t) .
\end{gathered}
$$

ke denotes drug clearance. $K$ and $L$ are transport coefficients from the plasma to the interstitium and from the interstitium to lymphatic vessels, respectively. $I(t)$ denotes the amount of drugs injected into plasma:

$$
I(t)=\left\{\begin{array}{l}
1, t=0 \\
0, t>0 .
\end{array}\right.
$$

The initial conditions are given by

$$
C_{P}(0)=0, \quad C_{e}(0)=0 .
$$

After drugs enter the tumor, the interstitial flow influences the drug delivery as well as the diffusion effect. Here, we use the results of the blood perfusion descried before. Due to the 
Table 1: Baseline parameter values used for drug delivery simulations [19-21]. Parameter values with superscripts " $\mathrm{T}$," "N" represent the internal and external parameters of the tumor.

\begin{tabular}{lccc}
\hline Parameter & Value & Parameter & Value \\
\hline$k e\left(\mathrm{~s}^{-1}\right)$ & $1.33 \times 10^{-4}$ & $P_{\text {eff }}\left(10^{-6} \mathrm{~cm} / \mathrm{s}\right)$ & $1.91^{\mathrm{N}}$ \\
$K\left(10^{-10} \mathrm{~cm}^{3} / \mathrm{s} / \mathrm{M}\right)$ & 1.566 & & $14.9^{\mathrm{T}}$ \\
$L\left(10^{-9} \mathrm{~cm}^{3} / \mathrm{s} / \mathrm{M}\right)$ & 1.138 & $D\left(10^{-8} \mathrm{~cm}^{2} / \mathrm{s}\right)$ & $1.2^{\mathrm{N}}$ \\
$\beta$ & 0.1 & & $4.4^{\mathrm{T}}$ \\
$K_{a}\left(10^{-4} / \mathrm{M} / \mathrm{s}\right)$ & $0^{\mathrm{N}}$ & $K_{d}$ & $0^{\mathrm{N}}$ \\
& $1.33^{\mathrm{T}}$ & & $1.51^{\mathrm{T}}$ \\
\hline
\end{tabular}

high IFP, the convection effect in the tumor can be ignored [17]. The concentration of drugs $(d(x, y, t))$ in the tumor satisfies the diffusion equation

$$
\begin{aligned}
& \frac{\partial d(x, y, t)}{\partial t}+\nabla \cdot\left[R_{f} \vec{U}(x, y) d(x, y, t)\right] \\
& \quad=\nabla \cdot[D(x, y) \nabla d(x, y, t)]+\phi_{s}(x, y, t)-K_{a} \cdot d(x, y, t) \cdot\left[A_{0}-d_{B}(x, y, t)\right]+K_{d} \cdot d_{B}(x, y, t) \\
& \frac{\partial d_{B}(x, y, t)}{\partial t}=K_{a} \cdot d(x, y, t) \cdot\left[A_{0}-d_{B}(x, y, t)\right]-K_{d} \cdot d_{B}(x, y, t),
\end{aligned}
$$

where $R_{f}$ represents the relative velocity of drugs to the interstitial flow, $\vec{U}(x, y)$ is the velocity distribution of the flow and can be gotten from

$$
\vec{U}(x, y)=-K_{P} \cdot \nabla P_{i}
$$

(see [12]). $D(x, y)$ denotes the diffusion coefficient of drugs, $d_{B}(x, y, t)$ is the concentration of antigen-binding drugs, $K_{a}$ and $K_{d}$ represent the association rate and dissociation rate with antigens, and $\phi_{s}(x, y, t)$ is the source term given by Kedem-Katchalsky equation [18]

$$
\phi_{s}(x, y, t)=\Gamma(x, y) \cdot\left[C_{p}(t)-d(x, y, t)\right] \cdot A .
$$

Here, $A$ represents the generated vessel network, and $\Gamma(x, y)$ is the permeability across the capillary and is taken to be

$$
\Gamma(x, y)=\beta \cdot P_{\mathrm{eff}} \cdot \frac{S}{V^{\prime}}
$$

where $\beta$ is the corresponding coefficient and $P_{\text {eff }}$ denotes the effective permeability coefficient.

To solve the mathematical model, we use the FTBS method. The boundary condition is set to be $d(x, y, t)=0$ when the point $(x, y)$ is not in the computational domain. The initial conditions are $d(x, y, 0)=0$ and $d_{B}(x, y, 0)=0$.

The parameter values for simulation are in Table 1. 


\section{Results and Discussion}

In this paper, Figures 4 and 5 show the simulation results before and after antiangiogenic therapies. We choose six time points to see the drug distribution within two days after the injection. Figure 4 shows the uneven distribution of the concentration due to the blood vessel network. The concentration in the blood-vessel-rich region is higher. There are almost no vessels in the center, and the drug transports to this region only by diffusion. As a result, the concentration is relatively low. Without supplement, the concentration decreases as time goes. After four hours, the concentration gets smaller because of the drug clearance. Two days later, there are almost no drugs at all. The concentration of drug in solid tumor can be meliorated by normalization such as a lower IFP and a higher permeability. The drug delivery result in Figure 5 is much better, not only the maximum but also the area of drug distribution. Consequently it improves the outcome of chemotherapies.

In the paper, we ignore the change of the blood vessel network after antiangiogenic therapies. As a matter of fact, the structure, number, and distribution of the vasculature become different during the "vascular normalization window." In the further work, we will consider the factor. Besides, the sensitivity analysis of the parameters will be considered, so that we can elaborate on the effect of each parameter on the results. To do that, a quantitative measure should be defined to evaluate the effect of the antiangiogenic therapies, for example, the area covered by the drug.

The final goal of those combined therapies is to kill tumor cells, which makes it important to study how the tumor grows after therapies. Besides, studies have shown that antiangiogenesis-induced tumor cell starvation may enhance chemotherapeutic drug activity, despite a decrease in drug uptake. Balance can be found between them to improve the outcome of therapies if both are carefully scheduled.

\section{Acknowledgment}

The project was supported by the National Natural Science Foundation of China (10772751), Shanghai Leading Academic Discipline Project (B112).

\section{References}

[1] J. Folkman, "Therapeutic implications," New England Journal of Medicine, vol. 285, no. 21, pp. 11821186, 1971.

[2] R. K. Jain, "Normalization of tumor vasculature: an emerging concept in antiangiogenic therapy," Science, vol. 307, no. 5706, pp. 58-62, 2005.

[3] R. K. Jain, “Molecular regulation of vessel maturation," Nature Medicine, vol. 9, no. 6, pp. 685-693, 2003.

[4] R. K. Jain, "Barriers to drug delivery in solid tumors," Scientific American, vol. 271, no. 1, pp. 58-65, 1994.

[5] A. L. Harris, "Hypoxia: a key regulatory factor in tumor growth," Nature Reviews Cancer, vol. 2, no. 1, pp. 38-47, 2002.

[6] G. Helmlinger, F. Yuan, M. Dellian, and R. K. Jain, “Interstitial $\mathrm{pH}$ and $\mathrm{pO}_{2}$ gradients in solid tumors in vivo: high-resolution measurements reveal a lack of correlation," Nature Medicine, vol. 3, no. 2, pp. 177-182, 1997.

[7] J. L. Tatum et al., "Hypoxia: importance in tumor bioglogy, noninvasive measurement by imaging, and value of its measurement in the management of cancer therapy," International Journal of Radiation Biology, vol. 82, no. 10, pp. 699-757, 2006. 
[8] R. K. Jain, R. T. Tong, and L. L. Munn, "Effect of vascular normalization by antiangiogenic therapy on interstitial hypertension, peritumor edema, and lymphatic metastasis: insights from a mathematical model," Cancer Research, vol. 67, no. 6, pp. 2729-2735, 2007.

[9] S. R. McDougall, A. R. A. Anderson, M. A. J. Chaplain et al., "Mathematical modeling of flow through vascular network: implications for tumor-induced angiogenesis and chemotherapy strategies," Bulletin of Mathematical Biology, vol. 64, pp. 673-702, 2002.

[10] D. Tee and J. DiStefano III, "Simulation of tumor-induced angiogenesis and its response to antiangiogenic drug treatment: mode of drug delivery and clearance rate dependencies," Journal of Cancer Research and Clinical Oncology, vol. 130, no. 1, pp. 15-24, 2004.

[11] Z. Gaiping, G. Hao, W. Jie et al., “2D numerical simulation of effect of antiangiogenic factors Angiostatin and Endostatin on tumor-induced angiogenesis," Journal of Medical Biomechanics, vol. 21, no. 4, pp. 272-279, 2006.

[12] J. Wu, Q. Long, S. Xu, and A. R. Oadhani, "Study of tumor blood perfusion and its variation due to vascular normalization by anti-angiogenic therapy based on 3D angiogenic microvasculature," Journal of Biomechanics, vol. 42, no. 6, pp. 712-721, 2009.

[13] B. Rippe and B. Haraldsson, "Capillary permeability in rat hindquaters as determined by estimations of capillary reflection coefficients," Acta Physiologica Scandinavica, vol. 127, no. 3, pp. 289-303, 1986.

[14] J. A. Tyrrell, V. Mahadevan, R. T. Tong, E. B. Brown, R. K. Jain, and B. Roysam, "A 2-D/3-D modelbased method to quantify the complexity of microvasculature imaged by in vivo multiphoton microscopy," Microvascular Research, vol. 70, no. 3, pp. 165-178, 2005.

[15] R. T. Tong, Y. Boucher, S. V. Kozin et al., "Vascular normalization by vascular endothelial growth factor receptor 2 blockade induces a pressure gradient across the vasculature and improves drug penetration in tumors," Cancer Research, vol. 64, pp. 3731-3737, 2004.

[16] C. Jinfeng, L. Jie, Z. Hongyi et al., "Research on the effect of microenvironment of tumor blood perfusion under anti-angiogenesis therapy," Progress in Biomedical Engineering, vol. 3, pp. 125-130, 2010 (Chinese).

[17] R. K. Jain, "Determinants of tumor blood flow: a review," Cancer Research, vol. 48, no. 10, pp. 26412658, 1988.

[18] O. Kedem and A. Katchalsky, "Thermodynamic analysis of permeability of biological membranes to non-electrolytes," Biochimica et Biophysica Acta, vol. 27, no. C, pp. 229-246, 1958.

[19] L. T. Baxter and R. K. Jain, "Transport of fluid and macromolecules in tumors. I. Role of interstitial pressure and convection," Microvascular Research, vol. 37, no. 1, pp. 77-104, 1989.

[20] L. T. Baxter and R. K. Jain, "Transport of fluid and macromolecules in tumors. II. Role of heterogeneous perfusion and lymphatics," Microvascular Research, vol. 40, pp. 246-263, 1990.

[21] L. T. Baxter and R. K. Jain, "Transport of fluid and macromolecules in tumors. III. Role of binding and metabolism," Microvascular Research, vol. 41, no. 1, pp. 5-23, 1991. 


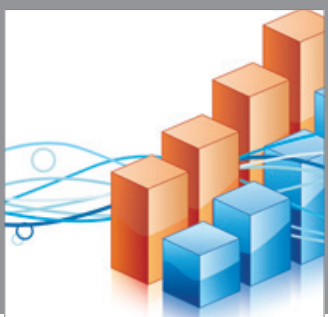

Advances in

Operations Research

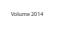

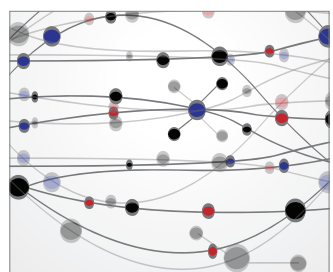

\section{The Scientific} World Journal
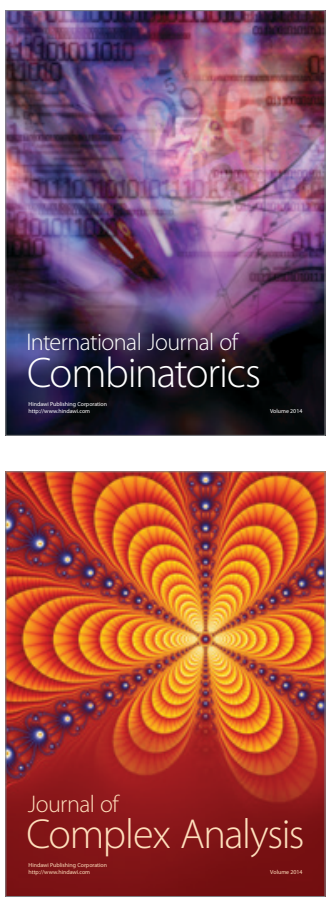

International Journal of

Mathematics and

Mathematical

Sciences
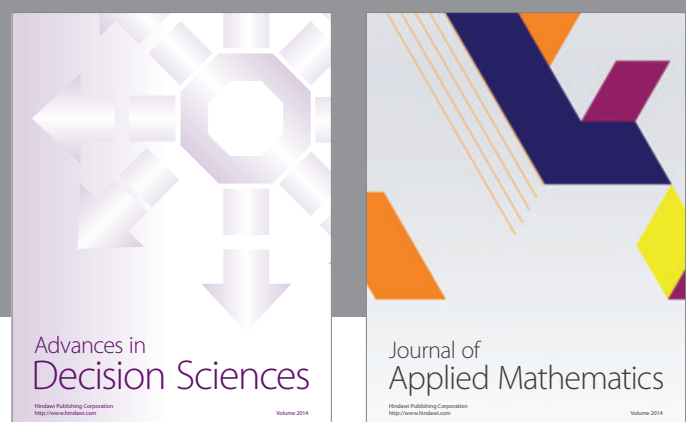

Journal of

Applied Mathematics
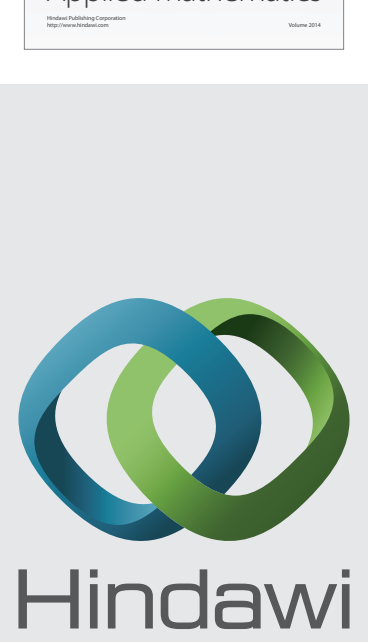

Submit your manuscripts at http://www.hindawi.com
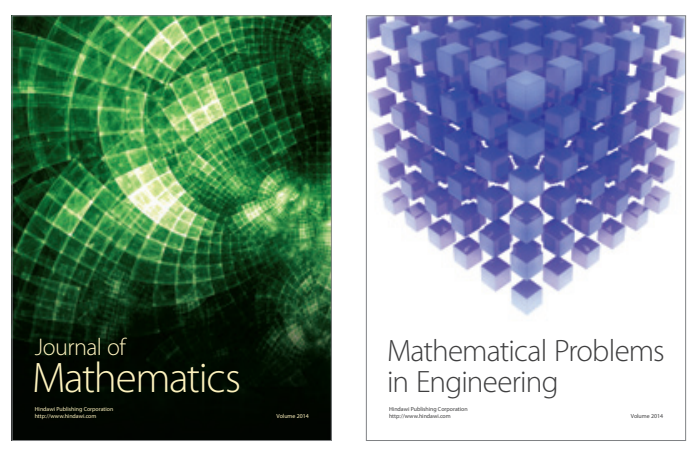

Mathematical Problems in Engineering
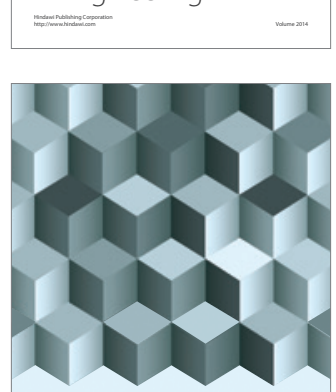

Journal of

Function Spaces
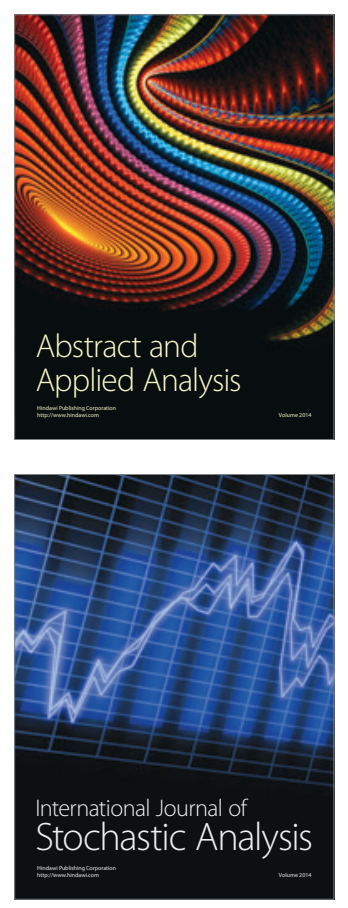

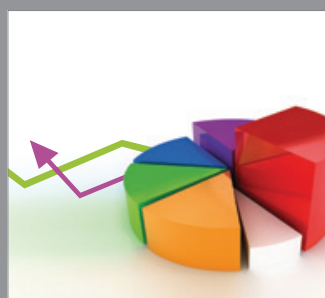

ournal of

Probability and Statistics

Promensencen
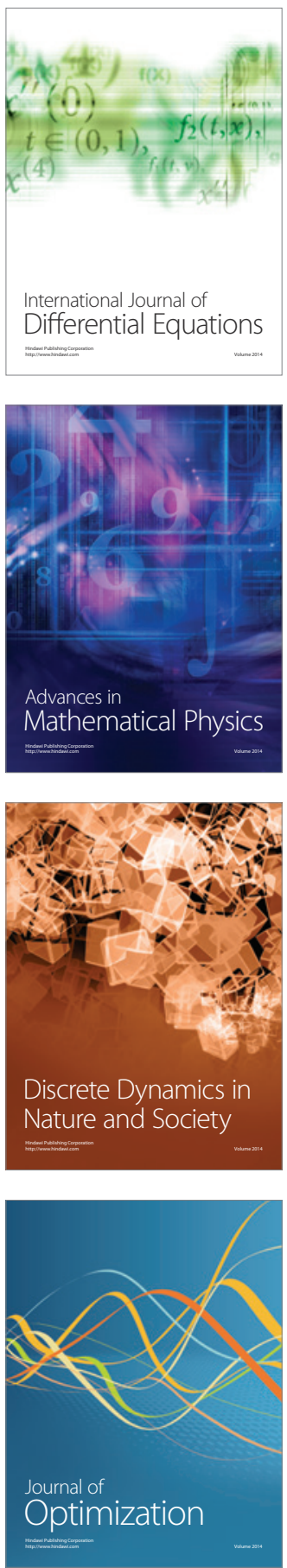